\section{Morgensteife erschwert Rheumakranken das Berufsleben enorm}

\author{
— Berufstätige Patienten mit Rheumatoi- \\ der Arthritis (RA) sind durch Morgensteifig- \\ keit ihrer Gelenke in vielen Fällen erheblich \\ beeinträchtigt. Das hat eine Befragung von \\ 1.061 RA-Patienten mit bekannter Morgen- \\ steifigkeit in elf europäischen Ländern erge- \\ ben. „Etwa die Hälfte der Befragten gab an, \\ noch aktiv im Arbeitsleben zu stehen", be- \\ richtete Prof. Frank Buttgereit von der Cha- \\ rité Berlin. \\ Mehr als jeder siebte der noch arbeitenden \\ RA-Patienten gab an, dass er im Monat vor \\ dem Interview wegen Morgensteifigkeit \\ teilweise nicht arbeiten konnte. In Deutsch- \\ land war es sogar jeder fünfte. Im Mittel
}

wurden 4,5 AU-Tage in Anspruch genommen. Jeder dritte kam in diesem Zeitraum zudem mindestens einmal zu spät zur Arbeit, weil wegen der steifen Gelenke die morgendliche Körperpflege und das Ankleiden mehr Zeit beanspruchte als eigentlich eingeplant.

Auch die Arbeit selbst wird vielfach durch die Morgensteifigkeit beeinträchtigt. Fast jeder zweite berufstätige RA-Patient gab an, dass die Morgensteifigkeit die Leistungsfähigkeit im Job im Mittel acht bis neun Stunden pro Woche beeinträchtige. In dieser Zeit arbeiten die Patienten um subjektiv geschätzte $40 \%$ weniger effektiv.

gvg

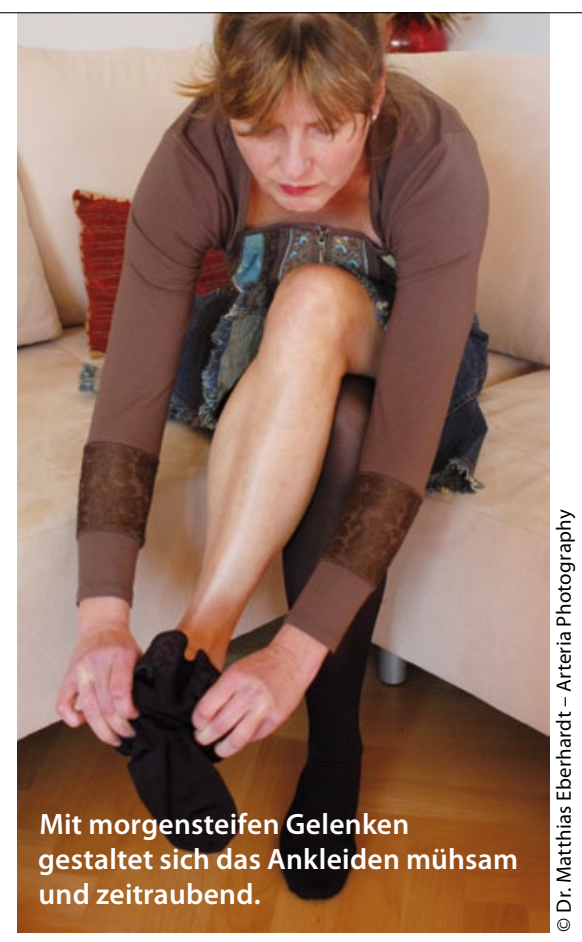

\title{
Gott sei Dank! Schlimme Rheuma-Gelenke sind vom Aussterben bedroht
}

— Die klassischen Zeichen der rheumatoider Arthritis (RA) verschwinden zusehens. Dies konstatierte Prof. Paul Emery von der Universität Leeds beim EULAR-Kongress 2012. Deformierte Hände oder versteifte Gelenke träten bei effektiver Therapie nicht mehr auf. Selbst subklinische Gelenkerosionen, die jahrzehntelang ein wichtiges diag-

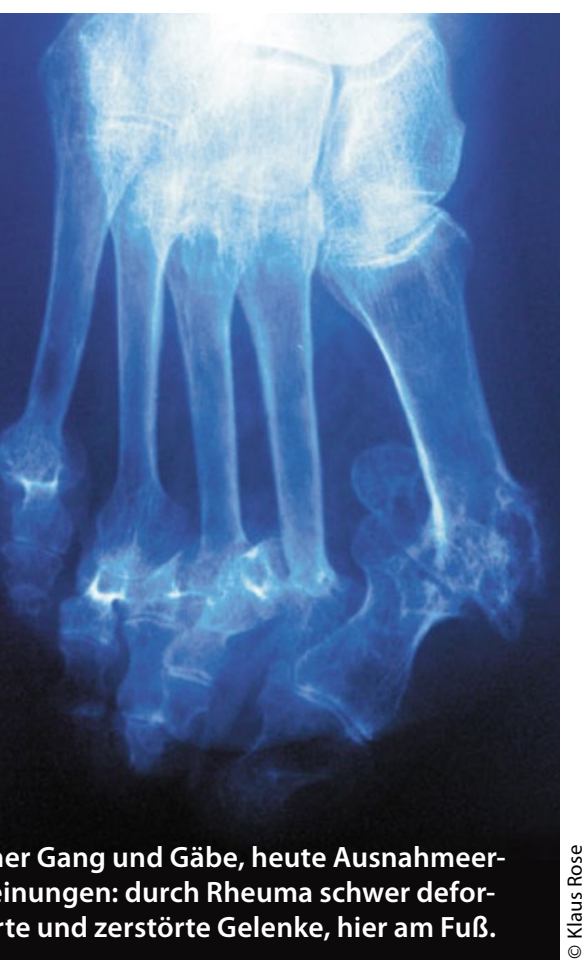

nostisches Kriterium der RA waren, seien heute - optimale Therapie vorausgesetzt fast schon Medizingeschichte.

Es liegt nah, diese Veränderungen zum Positiven mit den Biologika in Verbindung zu bringen. Das stimmt aber nur teilweise. Wichtiger sei ein konzeptioneller Wandel gewesen, so Emery: RA-Patienten werden heute nicht nur früher behandelt, sondern sehr viel engmaschiger überwacht. Ziel ist eine komplette Remission. Dies beinhaltet auch relativ rasche Therapiewechsel, wenn ein Patient nicht in Remission kommt.

Die Palette der Möglichkeiten ist bunt: Neben den klassischen Basistherapeutika (DMARDs), Glukokortikoiden und Anti-TNFalfa-Medikamenten gibt es inzwischen auch Wirkstoffe, die sich gegen B-Zellen oder Interleukin 6 richten. Für neue Biologika-Strategien gegen Interleukin 17 und 20 wurden bereits vielversprechende Ergebnisse vorgelegt. Prof. Josef Smolen vom AKH Wien trat allerdings ein wenig auf die Euphoriebremse und verwies auf die Behandlungsempfehlungen der Europäischen Rheumaliga, die für Ärzte nach wie vor die wichtigste Richtschnur sein sollten. Deren Quintessenz lautet: Biologika kommen am Ende, nicht am Anfang.

Standardtherapie zu Beginn blieben weiterhin klassische DMARDs und hier in erster Linie Methotrexat (MTX), betonte Smolen. Diese Wirkstoffe sollten idealerweise er- gänzt werden durch eine Kurzzeittherapie mit Glukokortikoiden. Erst wenn innerhalb von drei bis sechs Monaten keine Remission erreicht wird, folgt die Eskalation.

Der nächste Schritt sei in der Regel ein anderes DMARD oder die Kombination zweier DMARDs. „Nur wenn ungünstige prognostische Faktoren vorliegen, sieht die EULAR an dieser Stelle schon die Indikation für Biologika", verdeutlichte Smolen. Ungünstige Prognosefaktoren sind eine hohe Krankheitsaktivität, frühe Gelenkschäden oder hohe Level der Serummarker Rheumafaktor beziehungsweise Antikörper gegen citrullinierte Peptide (ACPA).

Insgesamt seien etwa $15 \%$ aller RA-Patienten tatsächlich Biologika-bedürftig, so Smolen. Mehrere von Wissenschaftlern initiierte Studien haben dieses eher zurückhaltende Vorgehen in letzter Zeit gestützt. So wurden in der randomisierten IDEA-Studie Patienten, die mit MTX und Infliximab therapiert wurden, mit solchen verglichen, die MTX und eine Einzelinjektion von 250 mg Methylprednisolon erhalten haben. „Nach einem Jahr gab es keine Unterschiede im radiologischen Ergebnis", berichtete Smolen.

Wird ein Biologikum eingesetzt, dann müssen sich Ärzte nach der derzeitigen Studienlage nicht allzu viele Gedanken darüber machen, welches sie wählen: Die klinischen und radiologischen Erfolgsraten seien alle sehr ähnlich, beteuerte Smolen. 